\title{
Daily measurements of the mean magnetic field of the Sun, 1968-2001: Anomalous distribution?
}

\begin{abstract}
V. A. Kotov ${ }^{\star}$
Crimean Astrophysical Observatory, Nauchny, Crimea 98409

Isaak Newton Institute of Chile, Crimean Branch

Received 4 December 2002 / Accepted 19 February 2003

Abstract. The mean magnetic field (MMF) of the Sun was measured in 1968-2001 by four Babcock magnetographs: of the Crimean Astrophysical Observatory, CrAO, of the Mount Wilson Observatory, MWO, of the Wilcox Solar Observatory, WSO, and of the Sayan Solar Observatory, SSO (in all nearly 13 thousand daily records). The MMF strengths recorded by these instruments on the same day, often deviate substantially from each other; this can hardly be explained by purely instrumental/solar causes alone. It is pointed out that $(a)$ each magnetograph represents a linear electro-optical device detecting diminutive, $\sim 10^{-5}-10^{-4}$, Zeeman circular polarization of a solar spectral line, with $(b)$ no essential nonliner effects are expected, and $(c)$ the observed MMF daily values must be normally distributed around a zero mean. The actual MMF distribution appears to be quite normal for records of the CrAO and SSO. Those of the MWO and WSO, however, deviate remarkably from the normal. The exact physical nature of this abnormity is unknown. It is suggested that the true cause might be connected with $(a)$ the use of an image-slicer (at MWO and WSO), (b) entanglement of photons, $(c)$ the statistical origin of light itself (due to the principle of uncertainty: "the instrument inevitably influences the output"), and $(d)$ some instrumental/solar causes of poorly known origin and action.
\end{abstract}

Key words. Sun: magnetic fields

\section{Introduction}

Significant discrepancies between measurements of the magnetic field of the Sun's photosphere performed by different Babcock solar magnetographs and using various spectral lines, including those with different Lande factors $g$, are frequently observed and not yet clearly understood (Howard \& Stenflo 1972; Stenflo 1973; Scherrer et al. 1977a; Kotov et al. 1998a; Demidov et al. 2002). It is widely accepted that discrepancies are caused mainly by the so-called "saturation" of the magnetograph signal due to the presence of fine structure $1.5-\mathrm{kG}$ magnetic elements in the photosphere.

The appropriate way to throw more light on the problem seems to be observations of the same magnetic source, the Sun, by different instruments: simultaneously and in the same spectral line. In the present study the Sun will be a "magnetic standard" for Zeeman polarization measurements by several solar magnetographs.

Over the last 34 years systematic records of the mean magnetic field (MMF, B) of the Sun-as-a-star were carried out by four institutes: the Crimean Astrophysical Observatory (CrAO), the Wount Wilson Observatory (MWO), the Wilcox

\footnotetext{
^ e-mail: vkotov@crao.crimea.ua
}

Solar Observatory (WSO) and the Sayan Solar Observatory (SSO, Irkutsk). In all, nearly 13 thousand MMF daily values were collected and published from 1968 through 2001 (Scherrer et al. 1977a,b; Grigoryev \& Demidov 1987; Kotov et al. 1998a,b).

During MMF observations a Babcock magnetograph records the longitudinal Zeeman effect of a Fraunhofer absorption line (Fe I $\lambda 525.02 \mathrm{~nm}, g=3$ ) in integrated solar light. The limb darkening enters in $B$ as a weighting function, together with several other factors, close to unity, caused by solar rotation, red shift of a spectral line to solar limb, correlations of line profile with brightness and Doppler shift, possible instrumental vignetting etc. The $B$ value - for a given day represents an average strength of all photospheric fields. The most significant contribution to MMF comes from large-scale background fields of the "quiet" photosphere (Scherrer 1973; Demidov et al. 2002).

The MMF changes due to the Sun's rotation and time evolution of the sector structure of large-scale fields. Daily variations of MMF appear to be usually rather small, so that one may consider MMF measurements, performed by different instruments during the same UT-day, to be "simultaneous". At the present time we can analyse about 13 thousand MMF daily 
Table 1. The summary of MMF data 1968-2001 (with no normalization).

\begin{tabular}{lccrcc}
\hline \hline Observatory & Years & $\Delta(\mathrm{G})$ & $N$ & $S(\mathrm{G})$ & $\mathrm{k}$ \\
\hline CrAO & $1968-2001$ & 0.15 & 1945 & 0.67 & 0.94 \\
MWO & $1970-1982$ & 0.07 & 2457 & 0.67 & 0.94 \\
WSO & $1975-2001$ & 0.05 & 7952 & 0.41 & 1.54 \\
SSO & $1982-1993$ & 0.12 & 313 & 0.77 & 0.82 \\
\hline In all & $1968-2001$ & - & 12667 & - & - \\
\hline
\end{tabular}

records obtained independently at four different sites. Many of them are simultaneous, being obtained on the same UT-day by at least two instruments. We are interested in correlations of MMF daily values measured for the "magnetic standard Sun" over 34 years by four different instruments.

\section{The MMF data 1968-2001}

The summary of the MMF measurements is given in Table 1 ( $N$ the number of daily values, $\Delta$ the formal typical error of a single record, $S$ standard deviation of a given data set, $k$ normalization factor). A special procedure was used at each observatory to fix the instrumental zero level with sufficient precision (Scherrer et al. 1977b; Grigoryev \& Demidov 1987; Kotov et al. 1998b). Positive $B$ corresponds to $N$ (nothern) polarity, and the quoted uncertainties everywhere mean a standard $\pm 1 \sigma$ error. No calibration or saturation factors were applied to original data sets.

To get the combined data series 1968-2001 in Sect. 7, the original $B$ values of a given observatory were multiplied by a factor $k=\langle S\rangle / S$, where $\langle S\rangle=0.63 \mathrm{G}$ is the average of four $S$ values (of four observatories, see Table 1). This is a widely accepted procedure to merge data sets in one analysis. As a result, we obtained the normalized MMF series with 12667 daily values and $S=0.63 \mathrm{G}$. Note however that all plots below are shown for original data sets, with no $k$ factors.

\section{Comparison of the CrAO and WSO data}

In the present study the linear regression parameters (in the usual form $y=a+b \times x$ ) were calculated for various MMF sample data. We shall consider then the correlation, $r$, and regression, $b$, coefficients only, neglecting small shifts in $a$. (The type of regression, $y \mid x$, means that $y$ is considered as a random variable, but $x$ represents a non-random one.)

Figure 1 compares the $\mathrm{CrAO}$ and the WSO data for a part of 2001, with standard deviations $0.58 \mathrm{G}$ and $0.29 \mathrm{G}$, respectively. Qualitatively, $B$ variations agree well with each other $(r=0.52)$; on average however $\mathrm{CrAO}|B|$ values are about twice as much as the corresponding WSO values. The agreement becomes better if one applies a factor 0.50 - the ratio of two standard deviations - to the CrAO values. Significant differences exceeding formal statistical errors $\Delta$ will, however, remain. For a linear regression of 57 pairs of data in Fig. 1 we get $b=0.25$. But several other plots of MMF measurements performed at the two observatories during other time spans revealed different parameters of regression (see, e.g., Fig. 2).
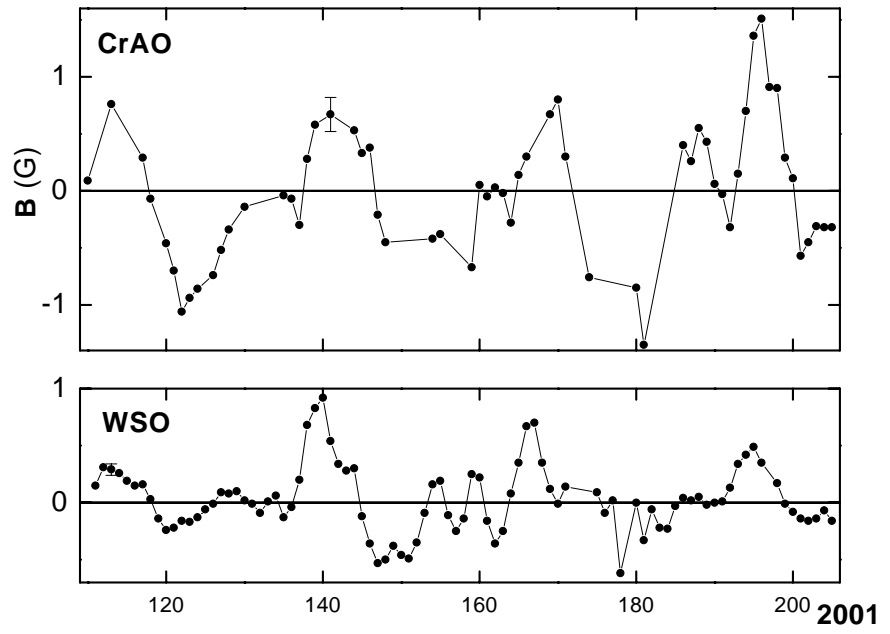

Fig. 1. Daily MMF values (dots connected by straight lines) according to the CrAO (top) and WSO (bottom) measurements from 20 April through 24 July, 2001. Horizontal axis gives consecutive day number of a year; the vertical bars indicate typical $\pm 1 \sigma$ errors.

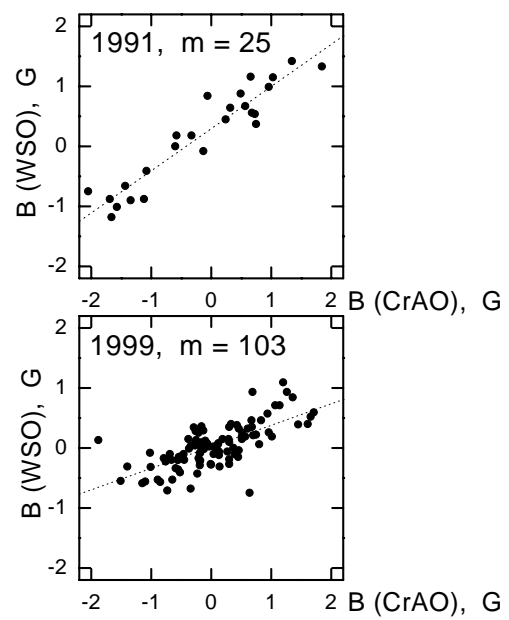

Fig. 2. Scatter-plot of WSO measurements vs. CrAO measurements performed in 1991, $m=25, r=0.94, b=0.70$ (top), and 1999, $m=103, r=0.70, b=0.36$ (bottom). The sloping dotted lines correspond to a linear regression analysis.

Table 2 shows that slope $b$ varies by more than twofold from year to year, with changes significant at more than $3 \sigma$ C.L. Similar variations of $b$ are observed also in many other samples of MMF data of four observatories (for cases of high, $>3 \sigma$, C.L. of linear $y \mid x$ dependence). Notice that such a strong variation of $b$ takes place for the same spectral line, 525.0, used by different magnetographs.

It is usually accepted that the main causes of discrepancies in slopes $b$ are instrumental: the shift of magnetograph zero-level, instability of electronics, vignetting of light beam, uncertainties of calibration, of adjustment of optic elements and electro-optical modulator, thermal effects and timevariable instrumental polarization (Scherrer 1973; Kotov 1977; Demidov 1996). The CrAO long-term magnetograph experience showed however that all the disturbancies of MMF measurements usually do not exceed roughly $5 \%$ to $15 \%$. But in reality the discrepancies between simultaneous MMF 
Table 2. Linear regression analysis of sample data ( $B$ is expressed in $\mathrm{G}, 1 \sigma$ errors of $a$ and $b$ are shown in brackets).

\begin{tabular}{llrcc}
\hline \hline $\begin{array}{l}\text { Observatories } \\
(x-y)\end{array}$ & Year & $m$ & $\begin{array}{c}\text { Equation } \\
(y=a+b \times x)\end{array}$ & $r$ \\
\hline MWO-WSO & 1979 & 191 & $y=+0.09(0.03)+0.28(0.03) \times x$ & 0.67 \\
$-"-$ & 1981 & 179 & $y=+0.06(0.04)+0.54(0.04) \times x$ & 0.77 \\
$-"-$ & 1982 & 175 & $y=+0.03(0.04)+1.20(0.07) \times x$ & 0.80 \\
\hline$-"-$ & $1975-1982$ & 1442 & $y=+0.02(0.01)+0.35(0.02) \times x$ & 0.58 \\
\hline CrAO-MWO & $1970-1976$ & 665 & $y=+0.03(0.03)+0.27(0.04) \times x$ & 0.26 \\
\hline CrAO-WSO & 1991 & 25 & $y=+0.30(0.07)+0.70(0.06) \times x$ & 0.94 \\
$-"-$ & 1999 & 103 & $y=+0.02(0.03)+0.36(0.04) \times x$ & 0.70 \\
$-"-$ & 2001 & 57 & $y=+0.05(0.03)+0.25(0.06) \times x$ & 0.52 \\
\hline$-"-$ & $1975-2001$ & 712 & $y=+0.08(0.01)+0.33(0.02) \times x$ & 0.65 \\
\hline SSO-MWO & 1982 & 32 & $y=-0.03(0.05)+0.47(0.04) \times x$ & 0.81 \\
\hline SSO-WSO & 1983 & 84 & $y=-0.06(0.04)+0.46(0.05) \times x$ & 0.81 \\
$-"-$ & 1993 & 28 & $y=-0.10(0.04)+0.72(0.15) \times x$ & 0.75 \\
\hline$-"-$ & $1982-1993$ & 256 & $y=-0.05(0.02)+0.51(0.03) \times x$ & 0.77 \\
\hline
\end{tabular}

measurements performed by different instruments are sometimes $50-100 \%$ or more. The detailed analysis of all reasonable sources of errors did not give clear answer as to the true nature of the discrepancies (Scherrer 1973; Duvall 1977; Kotov 1977; Grigoryev \& Demidov 1987; Demidov 1996).

\section{Entanglement of photons?}

Kotov \& Kotov (2002) supposed that these puzzling discrepancies might be attributed not to purely instrumental errors or fine structure of photospheric fields only (with the corresponding saturation effect; Howard \& Stenflo 1972; Stenflo 1973), but also to the quantum nature of light. Thus, the effect of entanglement of photons and the statistical nature of light itself must be taken into account for the interpretation of measurements of Zeeman polarization of small degrees.

Recently the effect of entanglement was proved experimentally in laboratories (Bouwmeester et al. 1999). According to quantum theory and the experiments, a pure quantum state e.g., a particular polarization - must be distributed between two objects, such as a pair of simultaneously generated photons, and they remain entangled even when separated. As a result, a measurement of one photon must influence the result of a measurement on its distant sibling. In our case, using an electro-optical modulator and polarizer in a magnetograph, we are recording polarization state of photons. The result must be inevitably influenced by the device - in accordance with splitting of a light beam by a polarizer and, perhaps, with the Heisenberg's uncertainty principle. (It is not yet known whether an entanglement is significant for processes of light emission in solar and stellar atmospheres and especially in the presence of a magnetic field; for a review of the "classic" problem see, e.g., Stenflo 1994.)

When measuring Zeeman polarization, however, one does not deal with a light beam of coherently created photons. The existence of the proposed effect in a magnetograph therefore is not yet proved and must be studied properly from theoretical and experimental points of view. But the uncertainty principle, in which physical nature is, in some sense, akin to entanglement, must operate in any case and might result in a significant distortion of the final polarization state of photons and, thus, in a distortion of the results of measurements of solar magnetic fields.

To get additional information on the nature of the enhanced scatter of daily MMF strengths we shall consider below the statistical distribution of MMF values.

\section{The CrAO and SSO distributions}

It is well known that virtually in every physical experiment the errors - or deviations from the mean value - show almost normal distribution. It is reasonable to expect therefore that if the Sun were a non-variable magnetic star, the measured MMF values would reveal a practically normal, or Gaussian, distribution around the mean. (The latter is almost zero for the total normalized MMF data series. A possible, but yet questionable, small systematic negative shift of $-0.02 \pm 0.01 \mathrm{G}$ - reported recently by Kotov et al. 2002 - is not significant for the present study.)

In fact however the real Sun - our standard magnetic "candle" - varies its mean field due to rotation and time evolution of background fields. These changes occur however within narrow limits, mostly from $-1 \mathrm{G}$ to $+1 \mathrm{G}$. For the long-term MMF series such changes manifest themselves as random walk and can thus result in an increase in the final scatter of $B$ values only. The classical reasoning about $B$ distribution therefore would be valid: it should be expected to be normal.

In Fig. 3a we plotted the distribution of the total set of the CrAO $B$ values $1968-2001$. It can be fairly well approximated by a normal distribution with a standard deviation $S_{0}=0.58 \mathrm{G}$. (Note that the actual $S$ value for the total $\mathrm{CrAO}$ data is equal to $0.67 \mathrm{G}$, see Sect. 2. There is a small, rather insignificant, excess of strong negative fields for $B \lesssim-1 \mathrm{G}$. Evidently, this excess results in an enhancement of the overall rms value, $S$, of the CrAO data with respect to $S_{0}$ in Fig. 3a.)

The Sayan distribution is shown in Fig. 3b. It may be also well approximated by a normal curve (with $S_{0}=0.83 \mathrm{G}$ which 


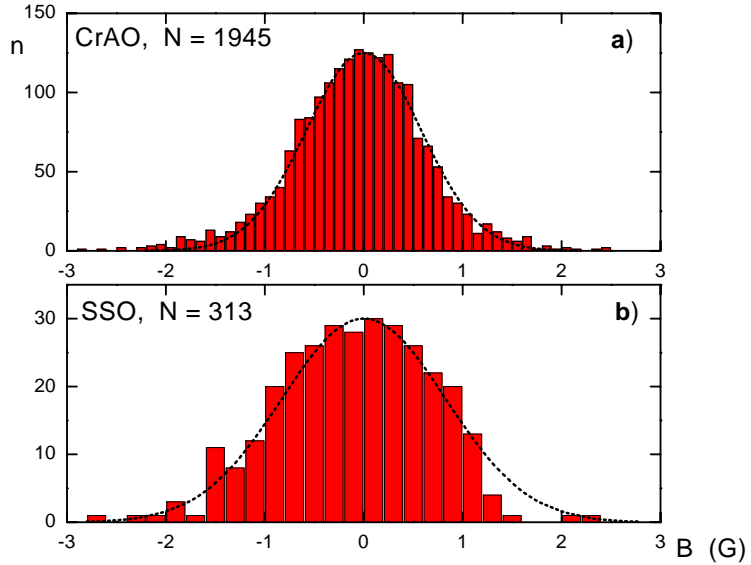

Fig. 3. a) Distribution of 1945 daily $B$ values recorded by the $\mathrm{CrAO}$ magnetograph from 1968 through 2001. The dotted line represents the best-fitted normal distribution ( $n$ the number of MMF records in each bin of the histogram). b) Same for Sayan measurements 1982-1993; $N=313$.

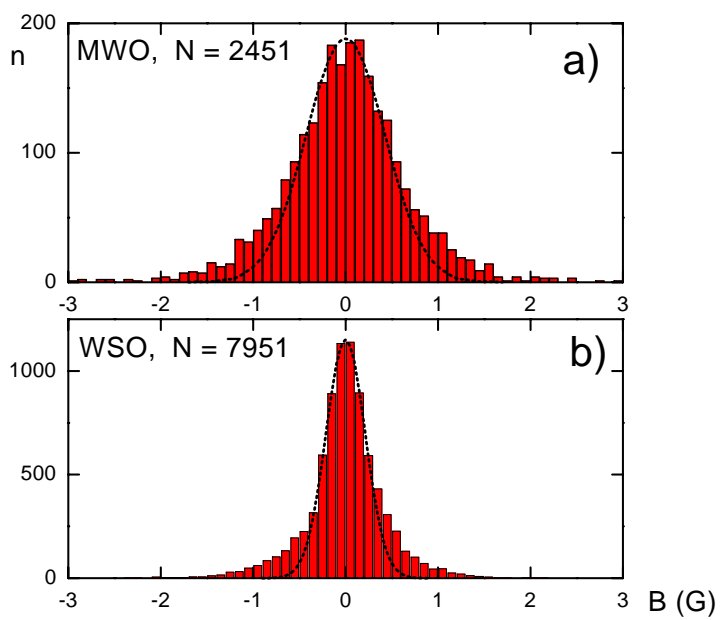

Fig. 4. a) Histogram of the MWO measurements 1970-1982 $(N=2451$; six MMF values, with intensity $|B|>3 \mathrm{G}$, were omitted from the plot). The dotted line corresponds to the normal distribution with $S_{0}=0.43 \mathrm{G}$, best-fitted for a central part of the data. b) Same for the WSO measurements 1975-2001 $\left(N=7951, S_{0}=0.21 \mathrm{G}\right.$; the highest value, $B=3.44 \mathrm{G}$, was omitted).

is a little bit higher than the original rms value $S=0.77 \mathrm{G}$ ). Some asymmetry of the actual distribution can be easily explained by scarcity of SSO data as compared with data sets of the other three observatories.

\section{The other two, amazing, histograms}

Figure 4a shows the distribution of the MWO data of 1970-1982 where the dotted line represents the normal distribution best-fit to the central portion of the data. The actual distribution significantly deviates from the normal one for intensities $|B| \gtrsim 0.5 \mathrm{G}$.

Figure $4 \mathrm{~b}$ gives a similar plot for the WSO data from 1975-2001 with $N=7951$. One can see again that for $|B| \gtrsim 0.4 \mathrm{G}$ this distribution deviates remarkably from the
Table 3. Comparison of rms values of three instruments.

\begin{tabular}{lcrcc}
\hline \hline Observatories & Years & $m$ & $\begin{array}{c}\Delta^{\prime} \\
(\mathrm{G})\end{array}$ & $\begin{array}{l}\Delta^{\prime \prime} \\
(\mathrm{G})\end{array}$ \\
\hline CrAO minus WSO & $1975-2001$ & 712 & 0.47 & 0.50 \\
MWO minus WSO & $1975-1982$ & 1442 & 0.60 & 0.64 \\
SSO minus WSO & $1982-1993$ & 256 & 0.51 & 0.62 \\
\hline
\end{tabular}

normal one. (Both distributions, according to the $\chi^{2}$ criterium, deviate from the normal one at more than $3 \sigma$ C.L.)

The trivial answer to the question about a plausible cause of the curious difference between two pairs of distributions, CrAO-SSO and MWO-WSO, could be a supposition that the $\mathrm{CrAO}$ and SSO have larger errors than MWO and WSO. This might be caused, for instance, by lower light flux used in the CrAO and SSO for MMF measurements, because the MWO and WSO spectrographs are supplied by image-slicers increasing light flux by more than 10 times (Scherrer et al. 1977b). In order to get a more definitive answer, however, the calibrations and real (normalized) errors of the instruments should be correctly accounted for.

\section{On calibration factors}

Our procedure for merging data from several observatories consists in reduction of original data sets to a common rms value, see Sect. 2. This is equivalent to multiplication of the original data by the normalization (calibration) factor $k$ listed in Table 1.

However, since we do not know the true, solar, $B$ value on any given day, the question about real errors, calibration factors or scatter in the MMF measurements is not simple. One of the more or less objective methods for evaluating differencies in calibration and, thus, in real errors, might be the following approach.

Let us assume that the most sensitive, WSO, measurements represent a good approximation of real $B$. We can make therefore a satisfactory estimation of scatter in simultaneous records of other three instruments - with respect to the "basic" WSO data (which mimic the "real magnetic Sun" in the best way) by calculating rms values $\Delta^{\prime}$ of deviations "CrAO minus WSO", etc. (It is assumed that differencies "WSO minus Sun" have the ad hoc value $\Delta^{\prime}=0$.) Note that for this procedure we use the normalized, 1968-2001, data series, with calibration differencies a priori accounted for. The results of computation are given in Table 3 where $\Delta^{\prime}$ means the rms deviation for normalized 1968-2001 data series and $\Delta^{\prime \prime}-$ the corresponding rms deviation reduced to original, non-normalized, data sets.

There are no major differences between $\Delta^{\prime}$ (or $\Delta^{\prime \prime}$ ) values of three data sets: the relative - with respect to the WSO data and thus to "the best-way approximated magnetic Sun" - rms deviations of $B$ values for the CrAO, MWO and SSO are nearly identical. One must conclude therefore that the difference between $B$ distributions (Fig. 3 versus Fig. 4) cannot be attributed to differences in accuracy (sensitivity) of MMF measurements. 


\section{Discussion}

During an MMF measurement each magnetograph represents a linear electro-optical device detecting tiny, $\sim 10^{-5}-10^{-4}$, Zeeman circular polarization in the wings of an absorption spectral line, with no essential nonliner effects - of purely instrumental origin - expected. (The above-quoted polarization corresponds to a $B$ intensity of the order of $0.05-0.50 \mathrm{G}$.)

The MMF changes polarity with a characteristic time scale of 6-14 days due to rotation and time evolution of 2- or 4-sector structures. Each MMF value is the average of a lot of fields of solar "quiet" regions (background fields) and a number of highly concentrated, sub-arcsec, flux tubes/filaments/fibrils/ropes (Stenflo 1973). The field strengths and sizes of all those magnetic elements are thought to be distributed randomly.

According to the current theory of field generation by a dynamo, magnetic elements and structures of the photosphere should be considered as by-products of turbulent, stochastic plasma motions of the solar convective zone; field elements must have therefore pure random statistics too. Notice also that we analyse MMF data collected by four instruments over many years - during a number of solar rotations and at various phases of 11-year cycle. The distribution of MMF values is therefore expected to be close to normal.

Indeed, let us suppose that magnetic elements in the photosphere have the same intensity (about $1.5 \mathrm{kG}$ ) but different polarities. The distribution of resultant MMF signals - as normalized averages of magnetic fluxes of all those elements - will be binomial with the mean zero (Brandt 1970). But for a large number of elements ("experiments"), the binomial distribution tends to be normal. And further, in accordance with the central limit theorem of statistics, one may expect that this distribution will not significantly differ from a normal one if one considers averages of a lot of elements of various random magnetic intensities, polarities and sizes. One should also stress that the $B$ value recorded simultaneously by various instruments, is expected to be - within the error limits and within the uncertainty of calibration - practically identical.

In fact, however, we see large differences, exceeding all reasonable limits of errors, between $B$ values recorded by different instruments. Moreover, the $B$ distributions of the two magnetographs, of the MWO and the WSO, differ remarkably from a normal distribution. Contrary to that, the $\mathrm{CrAO}$ and the SSO distributions look normal. The phenomenon of strong, highly concentrated magnetic ropes cannot explain discrepancies between the two pairs of instruments because the saturation effect must manifest itself more or less identically for all instruments considered.

We do not see any effects of purely instrumental/solar origin that can produce the remarkable deviation of the observed $B$ distributions from the normal one (Fig. 4). Plausibly, it arises from some non-linearity emerging in an instrument itself - at the light detection, perhaps. The true physical nature of the phenomenon is not yet known.

We point out only one "instrumental" (note: not solely instrumental) cause which might result in a difference between the two pairs of data sets. Namely, the MWO and WSO use image-slicers, but the CrAO and SSO do not. The image-slicer increases the light flux but introduces a distortion of the output due to entanglement of photons and/or simply due to the statistical nature of light. The main cause seems to be thus the act of measurement itself: in a Zeeman polarization measurement we underrate the capability of the instrument to distort the output.

We therefore postulate that

(a) the origin and physics of great "chaos" in MMF records relates, at least partly, to entanglement of photons and/or to the Heisenberg principle of uncertainty (which manifests not only for impulse/coordinate measurements but also for measurements of polarization state), and

(b) the physics of light itself and its interaction with the instrument ("an act of measurement") are assumed to be the main sources of non-linearity of the instrumental response resulting in appreciable abnormity of some $B$ distributions (Fig. 4).

This is only an hypothesis that certaintly requires further investigation. We believe that the truth can only be seen from within (from a measurement itself).

\section{Conclusion}

The MMF experiments discussed here give a clear illustration that the magnetic field measurement (of the Sun and other magnetic stars) cannot be divorced from the physics of light and from the act of measurement. We speculate thus that entanglement of polarized photons in a spectrograph, together with the operation of the uncertainty principle, can lead to a significant distortion of Zeeman polarization measurement and therefore to changes of magnetic field intensity recorded by a solar (stellar) magnetograph. The manifestation of both effects can be quite different for magnetographs of different optical and technical constructions. The best approximation to the true $B$ value can be obtained by the averaging of records performed with the use of several instruments and, perhaps, in various spectral lines (including those with differing Lande factors). It is our hope that future MMF measurements being actively persued at several sites will resolve the enigma of the striking $B$ distributions in Fig. 4.

On the other hand, it seems hard to imagine an appreciable presence of quantum effects of light when not single photons but huge numbers of them are collected. Certainly, many other instrumental/solar effects should be properly investigated (see, e.g., Stenflo 1994; Carroll \& Staude 2001) before it would be safe to state that pure solar/instrumental causes cannot explain the MMF discrepancies and abnormity of $B$ distribution.

Indeed, the line 525.0 is well known to be sensitive to temperature; this must result in rather different line profiles in various fine structures of the photosphere, such as granular interior and boundaries, fibrils etc. This would result in different "calibration curves" for transforming magnetograph records of those structures into field intensity. That means also different saturations that substantially depend on the exact position and width of the photometer exit slit in each magnetograph.

The integration over the solar disk averages the polarimetric data with a complex weighting function which could be significantly different for different devices, and a simple linear "calibration" would result in $B$ strengths differing from the 
potential result of actual spatial averaging of the field elements. Further complications might result from instrumental polarization, which is time variable and strongly dependent on telescope construction.

The consideration of the instrumental and solar effects requires detailed numerical simulations of Stokes line formation in the solar atmosphere (e.g., in a magneto-convection modelling; Stenflo 1994; Carrol \& Staude 2001) and special observations which of course cannot be made here and present a good task for future research.

Acknowledgements. The author thanks M. L. Demidov, J. T. Hoeksema and P. H. Scherrer for exchange of the MMF data, and J. Staude (the referee) for important and fair comments to the original manuscript which resulted in significant improvement of the note. Many fruitful discussions with V. I. Haneychuk, E. V. Ivanov, S. V. Kotov, J. O. Stenflo and T. T. Tsap on problems of solar magnetism and MMF observations, and the support from INTAS (grant No. 2000-840), are also greatly acknowledged.

\section{References}

Bouwmeester, D., Pan, J.-W., Mattle, K., et al. 1999, Nature, 390, 575 Brandt, S. 1970, Statistical and Computational Methods in Data Analysis (Amsterdam: North Holland Publ.)
Carroll, T. A., \& Staude, J. 2001, in Solar Encounter: The First Solar Orbiter Workshop, ESA SP-493, 183

Demidov, M. L. 1996, Sol. Phys., 164, 381

Demidov, M. L., Zhigalov, V. V., Peshcherov, V. S., \& Grigoryev, V. M. 2002, Sol. Phys., 209, 217

Duvall, T. L., Jr. 1977, Stanford Univ. Inst. Plasma Res. Rep., N 724

Grigoryev, V. M., \& Demidov, M. L. 1987, Sol. Phys., 114, 147

Howard, R., \& Stenflo, J. O. 1972, Sol. Phys., 22, 402

Kotov, V. A. 1977, Izv. Krym. Astrofiz. Obs., 56, 150

Kotov, V. A., Demidov, M. L., Haneychuk, V. I., \& Tsap, T. T. 1998a, Izv. Krym. Astrofiz. Obs., 94, 110

Kotov, V. A., Haneychuk, V. I., \& Tsap, T. T. 2002, Kinematika i fizika nebes. tel, 18, 205

Kotov, V. A., \& Kotov, S. V. 2002, in 1st Potsdam Thinkshop on Sunspots and Starspots, Poster Proc., ed. K. G. Strassmeier, \& A. Washuettl (Potsdam: AIP), 133

Kotov, V. A., Scherrer, P. H., Howard, R. F., \& Haneychuk, V. I. 1998b, ApJSS, 116, 103

Scherrer, P. H. 1973, Stanford Univ. Inst. Plasma Res. Rep., N. 554

Scherrer, P. H., Wilcox, J. M., Kotov, V., Severny, A. B., \& Howard, R. 1977a, Sol. Phys., 52, 3

Scherrer, P. H., Wilcox, J. M., Svalgaard, L., et al. 1977b, Sol. Phys., 54,353

Stenflo, J. O. 1973, Sol. Phys., 32, 41

Stenflo, J. O. 1994, Solar Magnetic Fields. Polarized Radiation Diagnostics (Dordrecht: Kluwer Acad. Publ.) 